\title{
Digitized-counterdiabatic quantum approximate optimization algorithm
}

\author{
P. Chandarana $\odot,{ }^{1, *}$ N. N. Hegade $\odot,{ }^{2,{ }^{*}}$ K. Paul $\odot,{ }^{2, \dagger}$ F. Albarrán-Arriagada, ${ }^{2}$ E. Solano,,${ }^{1,2,3,4, \star}$ \\ A. del Campo $\odot,{ }^{5,6}$ and Xi Chen $\odot^{1, \S}$ \\ ${ }^{1}$ Department of Physical Chemistry, University of the Basque Country UPV/EHU, Apartado 644, 48080 Bilbao, Spain \\ ${ }^{2}$ International Center of Quantum Artificial Intelligence for Science and Technology (QuArtist) \\ and Department of Physics, Shanghai University, Shanghai 200444, China \\ ${ }^{3}$ IKERBASQUE, Basque Foundation for Science, Plaza Euskadi 5, 48009 Bilbao, Spain \\ ${ }^{4}$ Kipu Quantum, Kurwenalstrasse 1, 80804 Munich, Germany \\ ${ }^{5}$ Department of Physics and Materials Science, University of Luxembourg, L-1511 Luxembourg, Luxembourg \\ ${ }^{6}$ Donostia International Physics Center, E-20018 San Sebastián, Spain
}

(Received 19 July 2021; accepted 14 January 2022; published 22 February 2022)

\begin{abstract}
The quantum approximate optimization algorithm (QAOA) has proved to be an effective classical-quantum algorithm serving multiple purposes, from solving combinatorial optimization problems to finding the ground state of many-body quantum systems. Since the QAOA is an Ansatz-dependent algorithm, there is always a need to design Ansätze for better optimization. To this end, we propose a digitized version of the QAOA enhanced via the use of shortcuts to adiabaticity. Specifically, we use a counterdiabatic (CD) driving term to design a better Ansatz, along with the Hamiltonian and mixing terms, enhancing the global performance. We apply our digitized-CD QAOA to Ising models, classical optimization problems, and the $P$-spin model, demonstrating that it outperforms the standard QAOA in all cases we study.
\end{abstract}

DOI: 10.1103/PhysRevResearch.4.013141

\section{INTRODUCTION}

Hybrid classical-quantum algorithms have the potential to unleash a broad set of applications in the quantum computing realm. The challenges involved in realizing fault-tolerant quantum computers have promoted the study of such hybrid algorithms, which proved to be relevant to modern noisy intermediate-scale quantum (NISQ) devices [1,2] with a few hundred qubits and limited coherence time. One notable example is that of the variational quantum algorithms (VQA), which is implemented by designing variational quantum circuits to minimize the expectation value for a given problem Hamiltonian. The VQA is advantageous given the fact that preparing a tunable circuit Ansatz is found to be difficult on a classical computer. It has already been widely applied in quantum chemistry [3-8], condensed matter physics [9-11], solving linear systems of equations [12], combinatorial optimization problems $[13,14]$, and several others $[15,16]$. Remarkably, one of the early implementations of the VQA was performed using photonic quantum processors [17], which prompted further theoretical progress [18-23].

\footnotetext{
*These authors contributed equally to this work.

${ }^{\dagger}$ koushikpa109@gmail.com

‡enr.solano@gmail.com

§chenxi1979cn@gmail.com
}

Published by the American Physical Society under the terms of the Creative Commons Attribution 4.0 International license. Further distribution of this work must maintain attribution to the author(s) and the published article's title, journal citation, and DOI.
The VQA has been demonstrated in superconducting qubits [3,6,18] and trapped ions [8,24,25].

One compelling outcome of the VQA is the development of the quantum approximate optimization algorithm (QAOA) [26], which provides an alternative for solving combinatorial optimization problems using shallow quantum circuits with classically optimized parameters. In the past few years, there has been a rapid development in QAOA-based techniques that have been applied not only for solving conventional optimization problems like MaxCut but also for solving ground state problems in different physical systems [25,27,28]. Improved versions of the QAOA, like ADAPT-QAOA [29] and DigitalAnalog QAOA [30] have also been reported recently. Like any combinatorial optimization problem, the QAOA depends on optimizing a cost function to obtain the desired optimal state corresponding to a $p$-level parametrized quantum circuit. In addition, the choice of the approximate trial state, from which the cost function is obtained, is crucial to the success of the QAOA. Generally, this is done by using quantum adiabatic algorithms (QAAs) which produce near-optimal results for large $p$ which is not suitable for current NISQ devices. Moreover, due to the requirement of large $p$, the cost of classical optimization increases, and the algorithms suffer from the problem of vanishing gradients and local minima [31-33].

Several studies have been reported in the past few years showing that high-fidelity quantum states can be prepared by assisting QAAs with additional driving interaction [34]. These studies establish that, for certain problems, the inclusion of additional driving terms can reduce the computational complexity and with it the circuit depth. These driving terms are usually calculated using methods developed under the umbrella of so-called shortcuts to adiabaticity [35,36], which 
have been introduced to improve the traditional quantum adiabatic processes, removing the requirement for slow driving [37]. Instances of these methods include counterdiabatic (CD) driving [38-40], fast-forward approach [41,42], and invariant-based inverse engineering $[43,44]$. Among them, CD driving is interesting and has been used to study fast dynamics [45-49], preparation of entangled states [50-53], adiabatic quantum computing [34,54], and quantum annealing [55-57].

In the context of the QAOA, the advantage of the introduction of $\mathrm{CD}$ driving is twofold. The $\mathrm{CD}$ driving decreases the circuit depth, while reducing the number of optimization parameters. On the other hand, it provides a better approximate trial state which is beneficial for finding the optimal target state. In this paper, we propose an algorithm, the Digitizedcounterdiabatic QAOA (DC-QAOA), which improves the performance of the conventional QAOA using CD driving. In this context, it is worthwhile to mention the work of Ref. [58], also inspired by CD driving techniques.

This paper is organized as follows. In Sec. II, we introduce the DC-QAOA and explain it in detail, comparing it with the quantum adiabatic evolution and the QAOA. In the following sections, we present a comparative study of the proposed DC-QAOA and the conventional QAOA method in the context of various physical systems. In Sec. III, we prepare the ground state of three different types of onedimensional (1D) Ising spin models, namely, the longitudinal field Ising model (LFIM), the transverse field Ising model (TFIM), and the Greenberger-Horne-Zeilinger (GHZ) state. In Sec. IV, we study classical optimization problems such as the MaxCut problem and the Sherrington-Kirkpatrick (SK) model, while in Sec. V, different variants of the $P$-spin model are considered. In doing so, we establish, by comparing the approximation ratios, that the DC-QAOA is advantageous compared to the QAOA for shallow quantum circuits. Finally, we conclude with a brief discussion in Sec. VI.

\section{DC-QAOA}

The conventional QAOA method can be viewed as a combination of two distinct parts: the quantum part consists of a parameterized circuit Ansatz, which is in turn complemented by a classical optimization algorithm to determine the parameters that minimize (maximize) a predefined cost function. The circuit Ansatz for the quantum part is governed by an annealing Hamiltonian:

$$
H_{a}(t)=[1-\lambda(t)] H_{\text {mixer }}+\lambda(t) H_{\text {prob }},
$$

where $\lambda(t) \in[0,1]$ is the annealing schedule for $t \in[0, T]$. Here, $H_{\text {mixer }}=\sum_{i} h_{i} \sigma_{i}^{x}$ is the mixing Hamiltonian that produces an equal (weighted) superposition state in the computational basis to begin with, whereas the desired final state is the ground state (or an eigenstate) of $H_{\text {prob }}$. In continuous annealing, the system evolves from the eigenstate of $H_{\text {mixer }}$ to the eigenstate of $H_{\text {prob }}$ through adiabatic evolution. The corresponding digital adiabatic circuit Ansatz can be designed using the trotterized time evolution operator $[59,60]$ :

$$
U(0, T) \approx \prod_{j=1}^{p} \prod_{m=1}^{M} \exp \left[-i H_{m}(j \Delta t) \Delta t\right],
$$

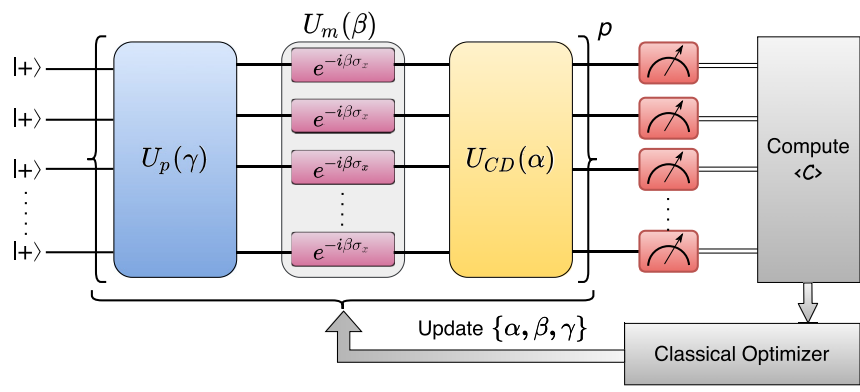

FIG. 1. Schematic diagram with circuit used for the Digitizedcounterdiabatic quantum approximate optimization algorithm (DCQAOA) having an additional CD term along with the Hamiltonian and mixing terms.

where we consider that $H_{a}(t)$ can be decomposed into $M k$-local terms, i.e., into terms $H_{m}(t)$ which have $k$-body interactions at most. Note that the $U(0, T)$ is a product of $p$ subunitaries, each corresponding to an infinitesimal propagation step $\Delta t$. An adiabatic evolution using $U(0, T)$ can always produce an exact target state at the cost of resorting to a large value of $p$. This can be translated to the language of the QAOA if one parameterizes $U(0, T)$ as

$$
\begin{aligned}
U(\boldsymbol{\gamma}, \boldsymbol{\beta})= & U_{m}\left(\beta_{p}\right) U_{p}\left(\gamma_{p}\right) U_{m}\left(\beta_{p-1}\right) U_{p}\left(\gamma_{p-1}\right) \ldots \\
& \ldots U_{m}\left(\beta_{1}\right) U_{p}\left(\gamma_{1}\right),
\end{aligned}
$$

where the evolution operators are $U_{m}\left(\beta_{p}\right)=\exp \left(-i \beta_{p} H_{\text {mixer }}\right)$ and $U_{p}\left(\gamma_{p}\right)=\exp \left(-i \gamma_{p} H_{\text {prob }}\right)$. Here, the annealing schedule is characterized by the discrete set of parameters $\left\{\beta_{p}, \beta_{p-1}, \ldots, \beta_{1}\right\}$ and $\left\{\gamma_{p}, \gamma_{p-1}, \ldots, \gamma_{1}\right\}$. Also, $(\boldsymbol{\gamma}, \boldsymbol{\beta}) \mathrm{de}-$ fines a $2 p$ parameter space that corresponds to the depth of the circuit Ansatz, and the cost function $F(\boldsymbol{\gamma}, \boldsymbol{\beta})$ is optimized classically to obtain an optimal parameter set $\left(\boldsymbol{\gamma}^{*}, \boldsymbol{\beta}^{*}\right)$, which produces the desired target state, i.e., $\left.\mid \psi\left(\boldsymbol{\gamma}^{*}, \boldsymbol{\beta}^{*}\right\rangle\right)$. Note that, in most cases, this target state is chosen to be the ground state of $H_{\text {prob. }}$.

As the case of adiabatic evolution, the QAOA requires large $p$ to obtain a near-optimal trial state, even with the assistance of the classical optimizer. In addition, the realization of $U(\boldsymbol{\beta}, \boldsymbol{\gamma})$ for an interacting many-body system for large $p$ becomes inefficient due to the large number of gates involved. In the DC-QAOA, we focus on improving the quantum part of the QAOA, by adding a variational parameter in each step, i.e.,

$$
U(\boldsymbol{\gamma}, \boldsymbol{\beta}) \rightarrow U(\boldsymbol{\gamma}, \boldsymbol{\beta}, \boldsymbol{\alpha}), \quad F(\boldsymbol{\gamma}, \boldsymbol{\beta}) \rightarrow F(\boldsymbol{\gamma}, \boldsymbol{\beta}, \boldsymbol{\alpha}) .
$$

The application of another parameter decreases the size of $p$ drastically. This additional parameter can be quantified as the inclusion of the CD driving term in the problem Hamiltonian. The resulting circuit Ansatz is shown in Fig. 1.

In general, $\mathrm{CD}$ driving amounts to using an additional control Hamiltonian in Eq. (1), required for suppressing nonadiabatic transitions [38-40,61]. This is especially effective for many-body systems with tightly spaced eigenstates. CD driving comes at a cost, as it generally involves nonlocal many-body interactions, and their exact specification of the CD Hamiltonian term requires access to the spectral properties of the driven system $[38,40,45]$. As a way out, variational 
approximations have been proposed to obtain the CD terms $[50,62,63]$. In this context, one can use the adiabatic gauge potential for finding an approximate CD driving without spectral information of the system $[63,64]$.

In the following sections, a pool of CD operators is defined using the nested commutator approach of the adiabatic gauge potential provided by Claeys et al. [65]:

$$
A_{\lambda}^{(l)}=i \sum_{k=1}^{l} \alpha_{k}(t) \underbrace{\left[H_{a},\left[H_{a}, \ldots\left[H_{a}\right.\right.\right.}_{2 k-1}, \partial_{\lambda} H_{a}]]] .
$$

Here, we considered up to the second order in the expansion of the nested commutator $l=2$, which gives rise to an operator pool $A=\left\{\sigma^{y}, \sigma^{z} \sigma^{y}, \sigma^{y} \sigma^{z}, \sigma^{x} \sigma^{y}, \sigma^{y} \sigma^{x}\right\}$, including solely local and two-body interactions. Note that the choice of this operator pool depends on $H_{a}(t)$ and may contain other operators depending on the problem Hamiltonian. However, $A$ contains every possible $C D$ operator that can be derived from the problem Hamiltonians, which is used in this paper. We chose the CD term as a combination of these operators for each system based on the success probability of the algorithm. For instance, the local CD driving term provides better success probability in the case of the TFIM and $P$-spin model; however, it is not suitable for solving the MaxCut Hamiltonian. The CD coefficients $\alpha_{k}$ are transformed into the additional variational parameter associated with the $C D$ driving. The addition of such a new free parameter increases the degrees of freedom, making it possible to reach broader parts of the Hilbert space of the Hamiltonian with a lower circuit depth than in the QAOA. Furthermore, as the DC-QAOA only requires the operator form of the $\mathrm{CD}$ driving combined with the additional set of parameters $\boldsymbol{\alpha}$, it eliminates the requirement of complex calculation of the CD coefficients. The DC-QAOA is also more flexible in regard to the boundary conditions than the CD evolution, which permits the application of the driving term even for one step only. Moreover, the operators can be chosen heuristically and according to the requirement of the system which is being studied.

Although there are several ways to define the cost function, we opt for the most convenient one, which is the energy expectation value of the problem Hamiltonian calculated for the trial wave function:

$$
F(\boldsymbol{\gamma}, \boldsymbol{\beta}, \boldsymbol{\alpha})=\left\langle\psi(\boldsymbol{\gamma}, \boldsymbol{\beta}, \boldsymbol{\alpha})\left|H_{\text {prob }}\right| \psi(\boldsymbol{\gamma}, \boldsymbol{\beta}, \boldsymbol{\alpha})\right\rangle,
$$

where $\psi(\boldsymbol{\gamma}, \boldsymbol{\beta}, \boldsymbol{\alpha})$ represents the approximate trial state produced by the digitized CD Ansatz. The efficiency of our algorithm can be measured in terms of the approximation ratio given by

$$
\mathcal{R}=\frac{F(\boldsymbol{\gamma}, \boldsymbol{\beta}, \boldsymbol{\alpha})}{E_{0}},
$$

where $E_{0}$ is the ground state energy of the system.

Classical optimization techniques are an integral part of variational algorithms, which help to find the optimal parameters that minimize the cost function. This paper mainly considers two optimization techniques, namely, Momentum Optimizer and Adagrad Optimizer, which are specific examples of stochastic gradient descent (SGD) algorithms. Momentum Optimizer is a variant of SGD in which a momentum term is added along with the gradient descent. The prime purpose of the momentum term is to increase the parameter update rate when gradients are in the same direction and decrease the update rate when gradients point in a different direction [66]. On the other hand, the main purpose of Adagrad Optimizer is to change the update rate based on the past descent results [67]. Adagrad has shown great improvements in the robustness of SGD [68]. These two classical optimization techniques work pretty well for the cases we consider. This is because these optimization routines have proven faster convergence than gradient descent. Moreover, some of the cases we study involve a large Hilbert space, which may lead to local minima in the energy landscape. In the presence of steep gradients, the use of these techniques proves beneficial. This problem dependence of the performance is shared with other optimization routines such as Nesterov Momentum, Adam, and AdaMax. An overview and comparison about challenges faced by the different types of gradient descent optimization, can be found in Ref. [69].

\section{ISING SPIN MODELS}

1D quantum Ising spin chains are the manifestation of the simplest many-body systems that are widely studied in existing quantum processors. Numerous computational problems can be mapped to find the ground state of the Ising-like Hamiltonians, which makes it suitable for benchmarking various quantum algorithms. The general form of the Hamiltonian of the $1 \mathrm{D}$ Ising spin model is given by

$$
H_{\mathrm{prob}}(\sigma)=-\sum_{\langle i, j\rangle} J_{i j} \sigma_{i}^{z} \sigma_{j}^{z}-\sum_{i} h_{i} \sigma_{i}^{z}-\sum_{i} k_{i} \sigma_{i}^{x},
$$

where $\sigma_{i}^{\delta}$ denotes the Pauli matrices at the $i$ th site, and $\langle i, j\rangle$ corresponds to the nearest-neighbor interaction with strength $J_{i j}$. The onsite interaction terms $h_{i}$ and $k_{i}$ represent the longitudinal and transverse fields, respectively. We consider the periodic boundary conditions so that our model describes a ring of interacting spins [70-72]. Note that three special cases can be retrieved from Eq. (8): (i) the LFIM when $k_{i}=0$, (ii) the TFIM when $h_{i}=0$, and (iii) a special case when both $k_{i}=0$ and $h_{i}=0$, for which the resulting ground state of $H_{\text {prob }}$ is the highly entangled GHZ state [73-76]. For simplicity, we choose the system to be homogeneous, i.e., $J_{i j}=J$ as well as $h_{i}=h_{z}$ and $k_{i}=h_{x}$. To prepare an equal superposition of the qubits, as a input of the circuit Ansatz, the mixing Hamiltonian is chosen as $H_{\text {mixer }}=\sum_{i} \sigma_{i}^{x}$. To implement the DC-QAOA, as mentioned in Sec. II, along with the problem and mixer Hamiltonian, we include the CD term to define the circuit Ansatz. The CD operator is chosen heuristically from the operator pool $A$. For instance, in the case of the LFIM, the ground state is ferromagnetic and constitutes a large energy gap with the first excited state for the chosen interaction strengths. In such cases, the local driving term $A_{t}=\sum_{i} \sigma_{i}^{y}$ can produce the ground state. On the other hand, the ground state of the TFIM is closely spaced with the nearby excited states, which makes the local driving term insufficient. Similarly, the local driving term is also not suitable for the GHZ state [61]. Instead, the second-order term $A_{t}=\sum_{i} \sigma_{i}^{z} \sigma_{i+1}^{y}$ is more likely to produce a better result. The unitary operator that represents the $\mathrm{CD}$ part 

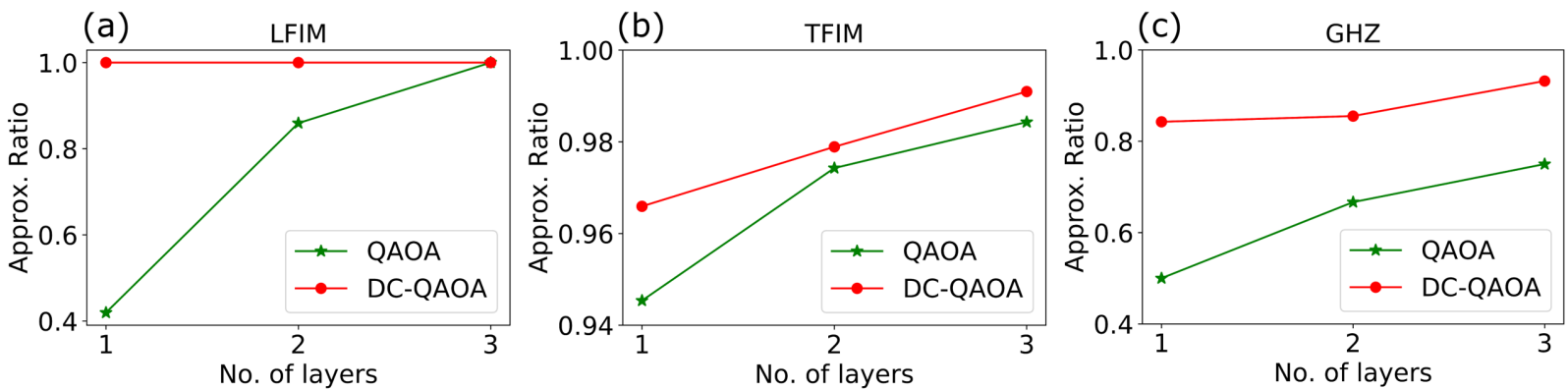

FIG. 2. Comparison of approximation ratios $(\mathcal{R})$ as a function of number of layers $(p)$ for three representative cases of Ising spin model. Green lines show results of the quantum approximate optimization algorithm (QAOA), whereas red lines show results of the digitized-counterdiabatic QAOA (DC-QAOA). (a) $\mathcal{R}$ variation of the longitudinal field Ising model (LFIM), where $J_{i j}=1, h_{i}=1$, and $k_{i}=0$. (b) The transverse field Ising model (TFIM), where $J_{i j}=1, h_{i}=0$, and $k_{i}=1$. (c) Preparation of the Greenberger-Horne-Zeilinger (GHZ) state, where $J_{i j}=1, h_{i}=0$, and $k_{i}=0$. System sizes for all cases were kept to $L=12$ qubits.

of the circuit Ansatz is given by

$$
U_{\mathrm{CD}}(\alpha)=\prod_{j=1}^{L} \exp \left[-i \alpha A_{t}^{q}\right],
$$

where $A_{t}^{q}$ represents the respective $q$-local CD operator chosen from the CD pool $A$. For instance, if $q=1$, then $A_{t}^{q}=\left\{A_{t}\right\}_{j}$, and if $q=2$, then $A_{t}^{q}=\left\{A_{t}\right\}_{j, j+1}$. The circuit is designed using the gate model of quantum computing, whereas the classical optimization is the SGD method. Figure 2 depicts the improvement obtained by the DC-QAOA over the traditional QAOA. In the simulation, we study a 12-qubit system, for which we compute $\mathcal{R}$ for different $p$ values. For the LFIM, as shown in Fig. 2(a), $\mathcal{R}=1$ even for $p=1$ with the DCQAOA, which constitutes considerable improvement over the QAOA, which requires $p=3$ to achieve unit $\mathcal{R}$. Hence, for Fig. 2(a), the number of variational parameters required to achieve unit $\mathcal{R}=1$ for the DC-QAOA is $3 p=3$, whereas for the QAOA, it is $2 p=6$. We also see that, for a lower number of layers, i.e., $p=1,2,3$, the DC-QAOA converges faster to the unit $\mathcal{R}$ than the QAOA. Furthermore, while the DC-QAOA shows better convergence at lower depths, for the TFIM and the GHZ states, the exact ground state can only be achieved with $p \geqslant L / 2$ layers. This effect can be attributed to the Lieb-Robinson bound [77,78] which forces the circuits for the TFIM and the GHZ state to scale linearly with the system size to achieve unit $\mathcal{R}$.

To compare the resource requirements, both classical and quantum, one can inspect two crucial elements of these methods. In the case of systems with nearest-neighbor interactions, the increase in circuit depth per layer by adding a CD term will be constant, and it depends on the CD term chosen. The circuit depth can be quantified as $d \times p$, and the CD driving increases it to $\left(d+d_{c d}\right) \times p$, where $d_{c d}$ represents the increment in depth per layer. For the LFIM, the CD term $\sigma_{y}$ gives $d_{c d}=1$, whereas for the TFIM, $d_{c d}=4$ for $\sigma_{z} \sigma_{y}$. On the other hand, the increase in parameter space due to the $\mathrm{CD}$ term is always from $2 p$ to $3 p$, making the DC-QAOA advantageous specifically for low $p$ values. In the limit of large $p$, the performances of the QAOA and the DC-QAOA become comparable for fixed system size.

\section{CLASSICAL OPTIMIZATION PROBLEMS}

Thus far, we have discussed the applications of the DCQAOA for finding the ground state of the Ising model and preparing entangled states. Combinatorial optimization problems are another set of problems that can be encoded in the ground state of a quantum Hamiltonian, diagonal in the computational basis. Here, we discuss the application of the DC-QAOA for solving combinatorial optimization problems, where the main objective is to find the optimal solution for a given classical cost function. MaxCut is one fundamental combinatorial optimization problem that has been solved using the QAOA.

For the MaxCut problem, let us consider a graph $G=$ $(V, E)$, where $V$ and $E$ are the vertex and edge sets, respectively. We consider a classical cost function $C(z)$ defined on binary strings $z=\left(z_{1}, z_{2}, \ldots, z_{n}\right)$ and aim at separating the vertices into two sets so that the number of edges cut by $C(z)$ is maximized. This maximizes the classical cost function:

$$
C(z)=\frac{1}{2} \sum_{(i, j) \in E} w_{i j}\left(1-z_{i} z_{j}\right)
$$

where $w_{i j}$ represents the edge weight between vertices $i$ and $j$. Depending on the sets that the vertices of each edge are in after the cut, binary values (either 0 or 1 ) are assigned to variables $z_{i}$ and $z_{j}$ corresponding to respective vertices. This situation can be encoded in the ground state of the problem Hamiltonian by mapping the binary variables to Pauli operators:

$$
H(\sigma)=\sum_{(i, j) \in E} J_{i j} \sigma_{i}^{z} \sigma_{j}^{z} .
$$

Note that Eq. (11) also belongs to the Ising class and is equivalent to Eq. (8) for GHZ states if only nearest-neighbor interaction is considered, which is the case of the two-regular MaxCut. Here, to verify the performance of our algorithm, we consider the unweighted $\left(w_{i j}=J_{i j}=1\right)$ three-regular MaxCut problem, with each vertex connected to three other vertices. The CD operator pool can be obtained from the NC expansion and is given by $A=\left\{\sigma^{z} \sigma^{y}, \sigma^{y} \sigma^{z}\right\}$. In Fig. 3(a), the approximation ratios $\mathcal{R}$ for different graph sizes with up to 14 vertices (qubits) are shown for a single layer $(p=1)$. We notice that, for small graph sizes, say, 4 qubits), the DC-QAOA 

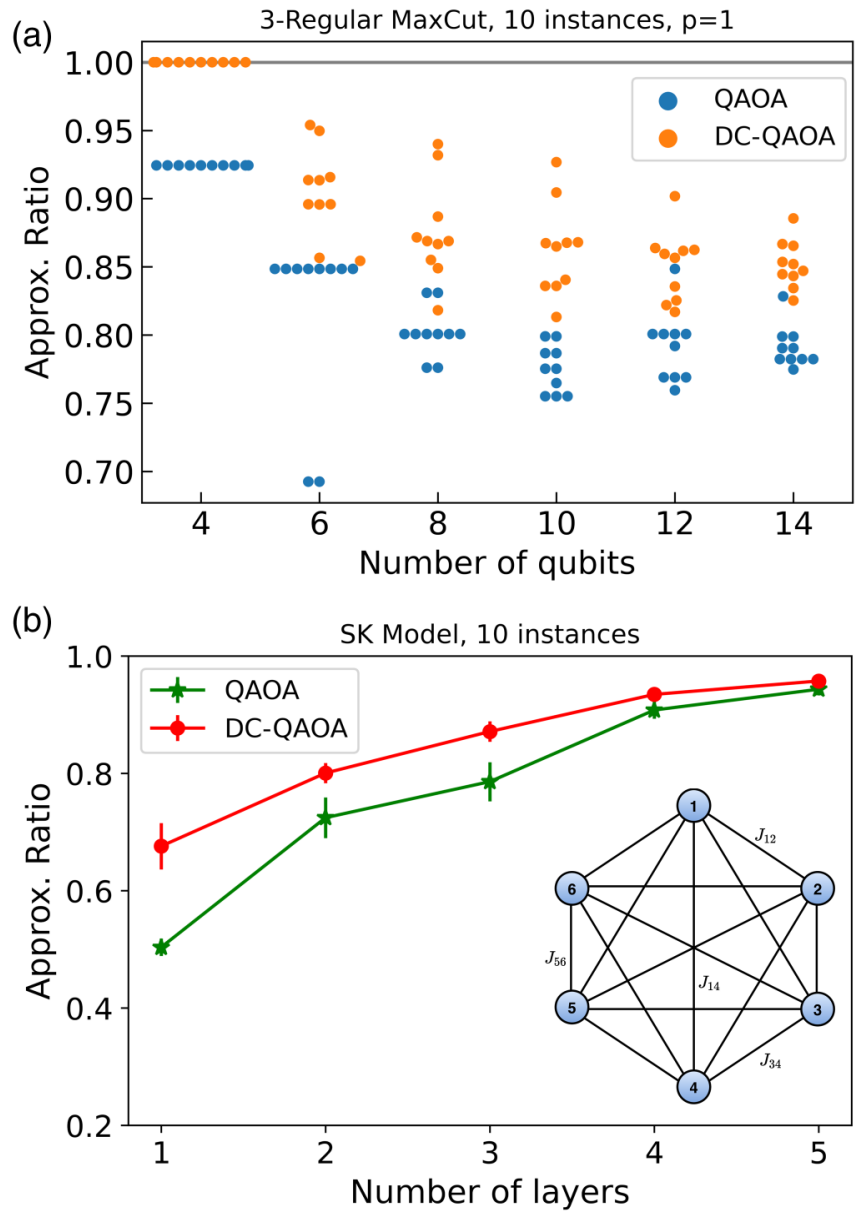

FIG. 3. Comparison of approximation ratios obtained for different graph size using the digitized-counterdiabatic quantum approximate optimization algorithm (DC-QAOA) and the QAOA. (a) Unweighted three-regular MaxCut for 10 randomly chosen instances. (b) Approximation ratio vs number of layers $(p)$ for the Sherrington-Kirkpatrick (SK) model with 6 qubits (vertices) is depicted. The green and red lines show the values of the QAOA and the DC-QAOA, respectively. On the right-bottom, a graph of 6 qubits with all-to-all connectivity is also shown. The results were obtained by considering 10 different randomly chosen instances of $J_{i j}$ values. Error bars represent the standard error.

is superior as it reaches unit $\mathcal{R}$. However, for a bigger graph, $\mathcal{R}$ decreases gradually while exceeding the performance of the QAOA. Although this can be improved for $p>1$ but for large depth in the DC-QAOA, the number of parameters for each step scales as $3 p$, so the landscape of the cost function most likely has a complicated form, and we expect to see the problem of vanishing gradients (Barren plateau). A detailed analysis is needed for $p>1$ in the DC-QAOA, which we leave for future work.

Interestingly, if $J_{i j}$ is chosen as random all-to-all two-body interactions, Eq. (11) represents the so-called SK model. The SK model is a classical spin model proposed by Sherrington and Kirkpatrick $[79,80]$ where $J_{i j}$ are interaction terms such that $J=\left\{\forall J_{i j}\right\}$ has zero mean and unit variance. For instance, they can be randomly chosen from the set $J=\{-1,1\}$ with probability $\frac{1}{2}$. The SK model is interesting for the DC-QAOA as it can be studied as a combinatorial search problem on a complete graph. The QAOA on the SK model has been extensively studied recently $[81,82]$. Here, 10 different instances of $J_{i j}$ values are considered in a system of $L=6$ spins. Note that the couplings $J_{i j}$ are nonuniform, and the CD term depends on the choice of $J_{i j}$. As this model involves similar interactions to that in the MaxCut problem, we chose the CD term from the same operator pool $A=\left\{\sigma^{z} \sigma^{y}, \sigma^{y} \sigma^{z}\right\}$, calculated from the nested commutator Ansatz. In fact, the $\mathrm{CD}$ term chosen for the SK model is $A_{t}=J_{i j} \sigma_{i}^{z} \sigma_{j}^{y}$, where the operators are applied to all the sites due to its all-to-all connectivity.

In Fig. 3(b), the approximation ratio $(\mathcal{R})$ is shown with respect to a varying number of layers $(p)$. We observe that $\mathcal{R}$ is higher for the DC-QAOA than the QAOA and that, as the number of layers increases, the DC-QAOA and the QAOA start to converge to the same value. This shows that the DCQAOA is efficient for instances where the circuit Ansatz is low layered. In fact, for low layers, although not giving the exact ground state, the DC-QAOA gives significantly enhanced $\mathcal{R}$. This could be advantageous as we can find optimal parameters which could be used as initial parameters for the high-layered QAOA.

\section{V. $P$-SPIN MODEL}

As a final benchmark, we consider the $P$-spin model, which is a long-range, exactly solvable, fully connected model [83-86]. The system Hamiltonian reads

$$
H=-\frac{1}{L^{P-1}}\left(\sum_{i=1}^{L} \sigma_{i}^{z}\right)^{P}-h \sum_{i=1}^{L} \sigma_{i}^{x} .
$$

While the ground state of the Hamiltonian in Eq. (12) is trivial, the presence of a quantum phase transition makes its preparation challenging by quantum annealing [83]. For $P=2$, this Hamiltonian exhibits a second-order phase transition, whereas a first-order phase transition occurs for $P \geqslant 3$, closing the energy gap exponentially with increasing system size. This has motivated proposals to change the first-order phase into a second-order phase transition by making the Hamiltonian nonstoquastic $[87,88]$. The nature of the ground state also depends on $P$. For odd $P$, the ground state is nondegenerate, while for even $P$, it has a twofold degeneracy with $Z_{2}$ symmetry, which makes the choice of the CD operator difficult [57]. We study the DC-QAOA in a 6 qubit $P$-spin model for the nontrivial case of $h \neq 0$ using local CD operator $A_{t}=\sum_{i} \sigma_{i}^{y}$. The QAOA and the DC-QAOA are compared for three different cases: $P=3, h=1 ; P=4, h=0$ and $P=4, h=1$ respectively. Figures 4 and 5 show the advantage obtained by the DC-QAOA for $P=4$ and 3, respectively. For $P=4$, $\mathcal{R}$ as a function of number of iterations is shown for $p=1$ for 10 random parameter initializations. We observe that, for a finite number of iterations, the DC-QAOA shows higher $\mathcal{R}$ values than the QAOA for both $h=0$ and 1 . It is evident that, in the case of $h=0$, the QAOA is highly dependent on the choice of initial parameters and lands into local minima in some instances. By contrast, the DC-QAOA shows unit $\mathcal{R}$ for 

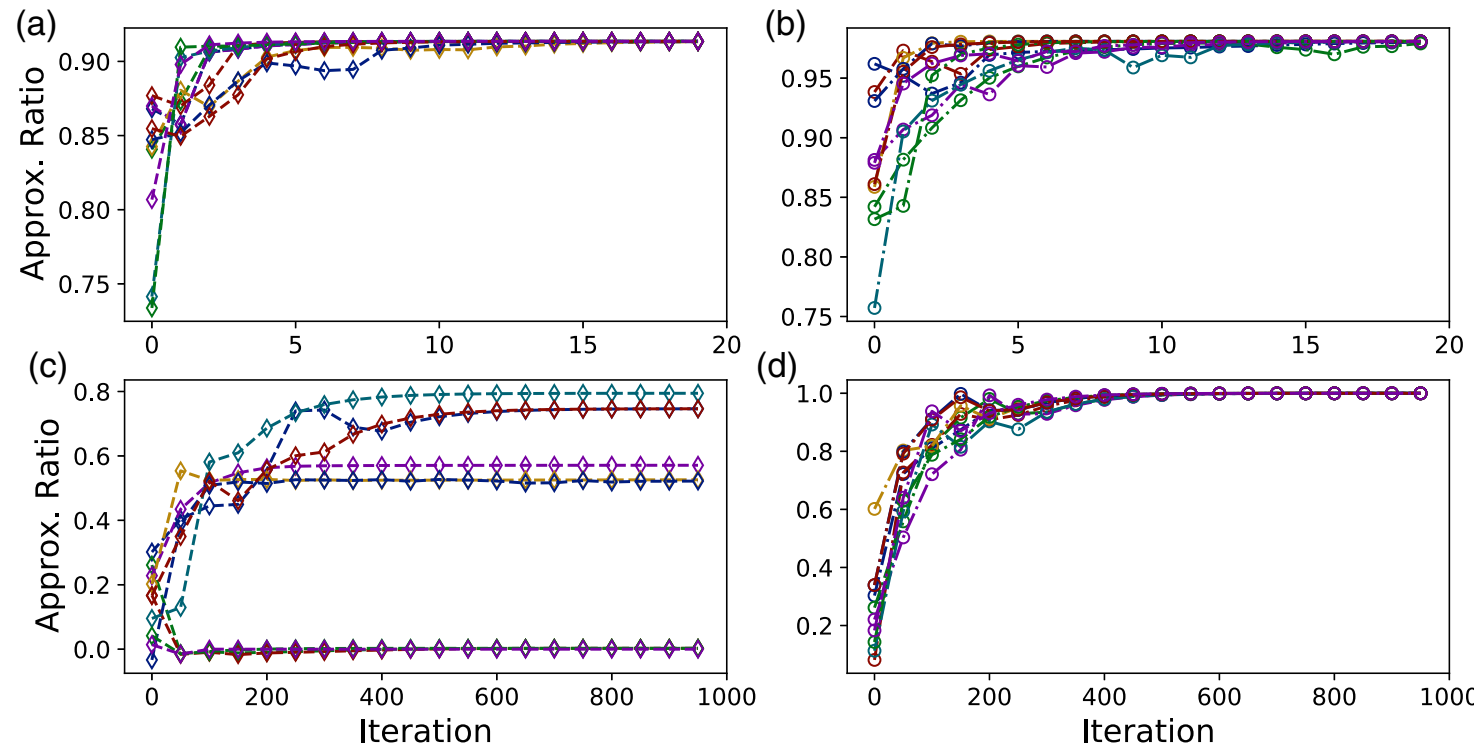

FIG. 4. Comparison of approximation ratio $(\mathcal{R})$ with respect to number of iterations for $P=4$ and $p=1$. (a) The quantum approximate optimization algorithm (QAOA) and (b) the digitized-counterdiabatic QAOA (DC-QAOA) results for $h=1$. The case with $h=0$ is shown for (c) the QAOA and (d) the DC-QAOA results. The system size is $L=6$. Each of the 10 random initial parameters chosen is represented by a color line.

every instance. For $P=3, h=1, \mathcal{R}$ is shown as a function of number of layers $(p=1,2,3)$ for 10 random initial parameters. As expected, for the DC-QAOA, $\mathcal{R}$ values end up close to unity even for $p=1$, and $\mathcal{R}$ values increase as the number of layers increases. However, this is not surprising for $P=3$, as the ground state is a product state making it favorable for the local CD operator. The more intriguing case is in Fig. 4(b), where the approximation ratio reaches close to unity for $p=1$ even when the ground state is degenerate. This occurs simply because the trial state converges to a particular one of the two due to the local CD driving. This is in contrast with the QAOA, which does not achieve the target state for $p=1$ in any case.

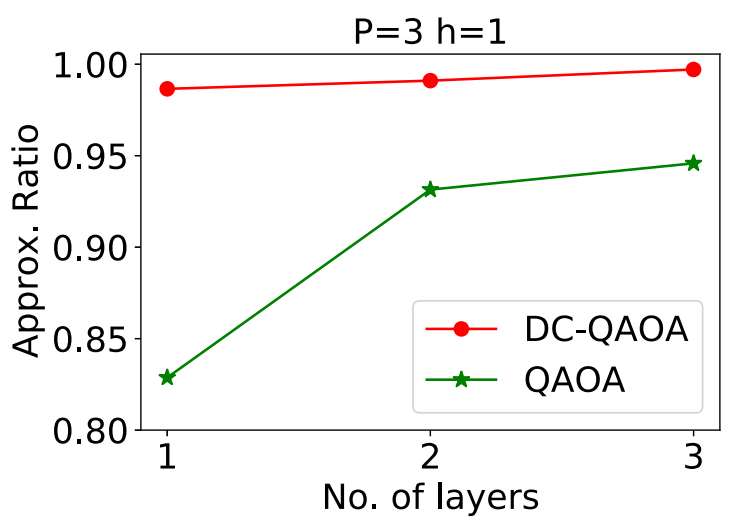

FIG. 5. Approximation ratio $(\mathcal{R})$ as a function of number of layers $(p=1,2,3)$ for $P=3$ and $h=1$. Green and red lines show the average results obtained from 10 random parameter initialization for the quantum approximate optimization algorithm (QAOA) and the digitized-counterdiabatic QAOA (DC-QAOA), respectively. Standard deviations are of the order of $10^{-2}$.

\section{DISCUSSION AND CONCLUSIONS}

We have introduced a quantum algorithm leveraging the strengths of shortcuts to adiabaticity for QAOAs. Specifically, we have formulated a variant of the QAOA using CD driving, called the DC-QAOA, and established its enhanced performance over the QAOA in finding ground states of different models. We benchmark our algorithm by considering various examples, starting with Ising spin models, preparing entangled states, classical optimization problems like MaxCut and the $\mathrm{SK}$ model, and the $P$-spin model. Including the CD term in the circuit Ansatz, the performance of the QAOA is enhanced. Results reveal that, for low-layered circuits, the DC-QAOA converges to the ground state faster than the state-of-the-art QAOA. Thus, adding a new free parameter in the form of a gate chosen from a predefined set (CD term) increases the performance of the algorithm for shorter circuit depths. Thus, the DC-QAOA turns out to be a preferable algorithm for circuits of shorter depth.

In conclusion, the DC-QAOA outperforms the QAOA for all the models we have studied. For high-depth circuits, the DC-QAOA can be applied for initial layers only to enhance the performance of the standard the QAOA. An interesting prospect would be to use the resulting optimal parameters from the low-depth DC-QAOA as the initial parameters of a high-depth QAOA to obtain the minima of the cost function efficiently. In this paper, we show that implementing principles of shortcuts to adiabaticity to enhance quantum algorithms has both fundamental and practical importance. The experimental realization of the DC-QAOA on real hardware offers an exciting prospect for further progress.

Note added in proof. As we finished this paper, we learned about the recent preprint devoted to the QAOA assisted by CD [89]. 


\section{ACKNOWLEDGMENTS}

This paper is supported by EU Future and Emerging Technologies (FET) Open Grants EPIQUS (899368) and Quromorphic (828826), the Basque Government IT986-16, the Spanish
Government PGC2018-095113-B-I00 (MCIU/AEI/FEDER, UE), projects QMiCS (820505) and OpenSuperQ (820363) of the EU Flagship on Quantum Technologies, NSFC (12075145), and STCSM (2019SHZDZX01-ZX04). X.C. acknowledges the Ramón y Cajal program (RYC-2017-22482).
[1] J. Preskill, Quantum computing in the NISQ era and beyond, Quantum 2, 79 (2018).

[2] K. Bharti, A. Cervera-Lierta, T. H. Kyaw, T. Haug, S. AlperinLea, A. Anand, M. Degroote, H. Heimonen, J. S. Kottmann, T. Menke, W.-K. Mok, S. Sim, L.-C. Kwek, and A. AspuruGuzik, Noisy intermediate-scale quantum (NISQ) algorithms, arXiv:2101.08448.

[3] J. I. Colless, V. V. Ramasesh, D. Dahlen, M. S. Blok, M. E. Kimchi-Schwartz, J. R. McClean, J. Carter, W. A. de Jong, and I. Siddiqi, Computation of Molecular Spectra on a Quantum Processor with an Error-Resilient Algorithm, Phys. Rev. X 8, 011021 (2018).

[4] H. R. Grimsley, S. E. Economou, E. Barnes, and N. J. Mayhall, An adaptive variational algorithm for exact molecular simulations on a quantum computer, Nat. Commun. 10, 3007 (2019).

[5] Y. Cao, J. Romero, J. P. Olson, M. Degroote, P. D. Johnson, M. Kieferová, I. D. Kivlichan, T. Menke, B. Peropadre, N. P. D. Sawaya, S. Sim, L. Veis, and A. Aspuru-Guzik, Quantum chemistry in the age of quantum computing, Chem. Rev. 119, 10856 (2019).

[6] A. Kandala, A. Mezzacapo, K. Temme, M. Takita, M. Brink, J. M. Chow, and J. M. Gambetta, Hardware-efficient variational quantum eigensolver for small molecules and quantum magnets, Nature 549, 242 (2017).

[7] Y. Nam, J.-S. Chen, N. C. Pisenti, K. Wright, C. Delaney, D. Maslov, K. R. Brown, S. Allen, J. M. Amini, J. Apisdorf, K. M. Beck, A. Blinov, V. Chaplin, M. Chmielewski, C. Collins, S. Debnath, K. M. Hudek, A. M. Ducore, M. Keesan, S. M. Kreikemeier et al., Ground-state energy estimation of the water molecule on a trapped-ion quantum computer, npj Quantum Inf. 6, 33 (2020).

[8] C. Hempel, C. Maier, J. Romero, J. McClean, T. Monz, H. Shen, P. Jurcevic, B. P. Lanyon, P. Love, R. Babbush, A. Aspuru-Guzik, R. Blatt, and C. F. Roos, Quantum Chemistry Calculations on a Trapped-ion Quantum Simulator, Phys. Rev. X 8, 031022 (2018).

[9] C. Cade, L. Mineh, A. Montanaro, and S. Stanisic, Strategies for solving the Fermi-Hubbard model on near-term quantum computers, Phys. Rev. B 102, 235122 (2020).

[10] S. Endo, I. Kurata, and Y. O. Nakagawa, Calculation of the Green's function on near-term quantum computers, Phys. Rev. Research 2, 033281 (2020).

[11] C. Bravo-Prieto, J. Lumbreras-Zarapico, L. Tagliacozzo, and J. I. Latorre, Scaling of variational quantum circuit depth for condensed matter systems, Quantum 4, 272 (2020).

[12] C. Bravo-Prieto, R. LaRose, M. Cerezo, Y. Subasi, L. Cincio, and P. J. Coles, Variational quantum linear solver, arXiv:1909.05820.

[13] X. Liu, A. Angone, R. Shaydulin, I. Safro, Y. Alexeev, and L. Cincio, Layer VQE: A variational approach for combinatorial optimization on noisy quantum computers, arXiv:2102.05566.
[14] G. Nannicini, Performance of hybrid quantum-classical variational heuristics for combinatorial optimization, Phys. Rev. E 99, 013304 (2019).

[15] E. R. Anschuetz, J. P. Olson, A. Aspuru-Guzik, and Y. Cao, Variational quantum factoring, arXiv:1808.08927.

[16] A. H. Karamlou, W. A. Simon, A. Katabarwa, T. L. Scholten, B. Peropadre, and Y. Cao, Analyzing the performance of variational quantum factoring on a superconducting quantum processor, npj Quantum Inf. 7, 156 (2021).

[17] A. Peruzzo, J. McClean, P. Shadbolt, M.-H. Yung, X.-Q. Zhou, P. J. Love, A. Aspuru-Guzik, and J. L. O'Brien, A variational eigenvalue solver on a photonic quantum processor, Nat. Commun. 5, 4213 (2014).

[18] P. J. J. O’Malley, R. Babbush, I. D. Kivlichan, J. Romero, J. R. McClean, R. Barends, J. Kelly, P. Roushan, A. Tranter, N. Ding, B. Campbell, Y. Chen, Z. Chen, B. Chiaro, A. Dunsworth, A. G. Fowler, E. Jeffrey, E. Lucero, A. Megrant, J. Y. Mutus et al., Scalable Quantum Simulation of Molecular Energies, Phys. Rev. X 6, 031007 (2016).

[19] J. R. McClean, J. Romero, R. Babbush, and A. Aspuru-Guzik, The theory of variational hybrid quantum-classical algorithms, New J. Phys. 18, 023023 (2016).

[20] J. R. McClean, M. E. Kimchi-Schwartz, J. Carter, and W. A. de Jong, Hybrid quantum-classical hierarchy for mitigation of decoherence and determination of excited states, Phys. Rev. A 95, 042308 (2017)

[21] P. K. Barkoutsos, J. F. Gonthier, I. Sokolov, N. Moll, G. Salis, A. Fuhrer, M. Ganzhorn, D. J. Egger, M. Troyer, A. Mezzacapo, S. Filipp, and I. Tavernelli, Quantum algorithms for electronic structure calculations: particle-hole hamiltonian and optimized wave-function expansions, Phys. Rev. A 98, 022322 (2018).

[22] J. Romero, R. Babbush, J. R. McClean, C. Hempel, P. J. Love, and A. Aspuru-Guzik, Strategies for quantum computing molecular energies using the unitary coupled cluster Ansatz, Quantum Sci. Technol. 4, 014008 (2018).

[23] J. Lee, W. J. Huggins, M. Head-Gordon, and K. B. Whaley, Generalized unitary coupled cluster wave functions for quantum computation, J. Chem. Theory Comput. 15, 311 (2019).

[24] Y. Shen, X. Zhang, S. Zhang, J.-N. Zhang, M.-H. Yung, and K. Kim, Quantum implementation of the unitary coupled cluster for simulating molecular electronic structure, Phys. Rev. A 95, 020501(R) (2017).

[25] G. Pagano, A. Bapat, P. Becker, K. S. Collins, A. De, P. W. Hess, H. B. Kaplan, A. Kyprianidis, W. L. Tan, C. Baldwin, L. T. Brady, A. Deshpande, F. Liu, S. Jordan, A. V. Gorshkov, and C. Monroe, Quantum approximate optimization of the long-range ising model with a trapped-ion quantum simulator, Proc. Natl. Acad. Sci. USA 117, 25396 (2020).

[26] E. Farhi, J. Goldstone, and S. Gutmann, A quantum approximate optimization algorithm, arXiv:1411.4028. 
[27] M. Willsch, D. Willsch, F. Jin, H. De Raedt, and K. Michielsen, Benchmarking the quantum approximate optimization algorithm, Quantum Inf. Proc. 19, 197 (2020).

[28] M. M. Wauters, G. B. Mbeng, and G. E. Santoro, Polynomial scaling of the quantum approximate optimization algorithm for ground-state preparation of the fully connected $p$-spin ferromagnet in a transverse field, Phys. Rev. A 102, 062404 (2020).

[29] L. Zhu, H. L. Tang, G. S. Barron, F. A. Calderon-Vargas, N. J. Mayhall, E. Barnes, and S. E. Economou, An adaptive quantum approximate optimization algorithm for solving combinatorial problems on a quantum computer, arXiv:2005.10258.

[30] D. Headley, T. Müller, A. Martin, E. Solano, M. Sanz, and F. K. Wilhelm, Approximating the quantum approximate optimisation algorithm, arXiv:2002.12215.

[31] J. R. McClean, S. Boixo, V. N. Smelyanskiy, R. Babbush, and $\mathrm{H}$. Neven, Barren plateaus in quantum neural network training landscapes, Nat. Commun. 9, 4812 (2018).

[32] M. Cerezo, A. Sone, T. Volkoff, L. Cincio, and P. J. Coles, Cost function dependent barren plateaus in shallow parametrized quantum circuits, Nat. Commun. 12, 1791 (2021).

[33] E. Grant, L. Wossnig, M. Ostaszewski, and M. Benedetti, An initialization strategy for addressing barren plateaus in parametrized quantum circuits, Quantum 3, 214 (2019).

[34] N. N. Hegade, K. Paul, Y. Ding, M. Sanz, F. AlbarránArriagada, E. Solano, and X. Chen, Shortcuts to Adiabaticity in Digitized Adiabatic Quantum Computing, Phys. Rev. Appl. 15, 024038 (2021).

[35] D. Guéry-Odelin, A. Ruschhaupt, A. Kiely, E. Torrontegui, S. Martínez-Garaot, and J. G. Muga, Shortcuts to adiabaticity: concepts, methods, and applications, Rev. Mod. Phys. 91, 045001 (2019).

[36] E. Torrontegui, S. Ibáñez, S. Martínez-Garaot, M. Modugno, A. del Campo, D. Guéry-Odelin, A. Ruschhaupt, X. Chen, and J. G. Muga, Chapter 2-Shortcuts to adiabaticity, Adv. At. Mol. Opt. Phys. 62, 117 (2013).

[37] K. Takahashi, Hamiltonian engineering for adiabatic quantum computation: Lessons from shortcuts to adiabaticity, J. Phys. Soc. Jpn. 88, 061002 (2019).

[38] M. Demirplak and S. A. Rice, Adiabatic population transfer with control fields, J. Phys. Chem. A 107, 9937 (2003).

[39] M. Demirplak and S. A. Rice, Assisted adiabatic passage revisited, J. Phys. Chem. B 109, 6838 (2005).

[40] M. V. Berry, Transitionless quantum driving, J. Phys. A: Math. Theor. 42, 365303 (2009).

[41] S. Masuda and K. Nakamura, Fast-forward problem in quantum mechanics, Phys. Rev. A 78, 062108 (2008).

[42] S. Masuda and K. Nakamura, Fast-forward of adiabatic dynamics in quantum mechanics, Proc. R. Soc. A 466, 1135 (2010).

[43] X. Chen, A. Ruschhaupt, S. Schmidt, A. del Campo, D. Guéry-Odelin, and J. G. Muga, Fast Optimal Frictionless Atom Cooling in Harmonic Traps: Shortcut to Adiabaticity, Phys. Rev. Lett. 104, 063002 (2010).

[44] X. Chen, E. Torrontegui, and J. G. Muga, Lewis-Riesenfeld invariants and transitionless quantum driving, Phys. Rev. A 83, 062116 (2011).

[45] A. del Campo, M. M. Rams, and W. H. Zurek, Assisted FiniteRate Adiabatic Passage Across a Quantum Critical Point: Exact Solution for the Quantum Ising Model, Phys. Rev. Lett. 109, 115703 (2012).
[46] A. B. Özgüler, R. Joynt, and M. G. Vavilov, Steering random spin systems to speed up the quantum adiabatic algorithm, Phys. Rev. A 98, 062311 (2018).

[47] K. Takahashi, Transitionless quantum driving for spin systems, Phys. Rev. E 87, 062117 (2013).

[48] A. Hartmann and W. Lechner, Rapid counter-diabatic sweeps in lattice gauge adiabatic quantum computing, New J. Phys. 21, 043025 (2019).

[49] F. Petiziol, B. Dive, F. Mintert, and S. Wimberger, Fast adiabatic evolution by oscillating initial Hamiltonians, Phys. Rev. A 98, 043436 (2018).

[50] T. Opatrný and K. Mølmer, Partial suppression of nonadiabatic transitions, New J. Phys. 16, 015025 (2014).

[51] F. Petiziol, B. Dive, S. Carretta, R. Mannella, F. Mintert, and S. Wimberger, Accelerating adiabatic protocols for entangling two qubits in circuit QED, Phys. Rev. A 99, 042315 (2019).

[52] H. Zhou, Y. Ji, X. Nie, X. Yang, X. Chen, J. Bian, and X. Peng, Experimental Realization of Shortcuts to Adiabaticity in a Nonintegrable Spin Chain by Local Counterdiabatic Driving, Phys. Rev. Appl. 13, 044059 (2020).

[53] Y. Ji, J. Bian, X. Chen, J. Li, X. Nie, H. Zhou, and X. Peng, Experimental preparation of Greenberger-Horne-Zeilinger states in an Ising spin model by partially suppressing the nonadiabatic transitions, Phys. Rev. A 99, 032323 (2019).

[54] N. N. Hegade, K. Paul, F. Albarrán-Arriagada, X. Chen, and E. Solano, Digitized-adiabatic quantum factorization, Phys. Rev. A 104, L050403 (2021).

[55] W. Vinci and D. A. Lidar, Non-stoquastic Hamiltonians in quantum annealing via geometric phases, npj Quantum Inf. 3, 38 (2017).

[56] K. Takahashi, Shortcuts to adiabaticity for quantum annealing, Phys. Rev. A 95, 012309 (2017).

[57] G. Passarelli, V. Cataudella, R. Fazio, and P. Lucignano, Counterdiabatic driving in the quantum annealing of the $p$-spin model: a variational approach, Phys. Rev. Research 2, 013283 (2020).

[58] J. Yao, L. Lin, and M. Bukov, Reinforcement Learning for Many-Body Ground State Preparation Based on CounterDiabatic Driving, Phys. Rev. X 11, 031070 (2021).

[59] D. Poulin, A. Qarry, R. Somma, and F. Verstraete, Quantum Simulation of Time-Dependent Hamiltonians and the Convenient Illusion of Hilbert Space, Phys. Rev. Lett. 106, 170501 (2011).

[60] M. Suzuki, Generalized Trotter's formula and systematic approximants of exponential operators and inner derivations with applications to many-body problems, Commun. Math. Phys. 51, 183 (1976).

[61] A. del Campo, Shortcuts to Adiabaticity by Counterdiabatic Driving, Phys. Rev. Lett. 111, 100502 (2013).

[62] H. Saberi, T. Opatrný, K. Mølmer, and A. del Campo, Adiabatic tracking of quantum many-body dynamics, Phys. Rev. A 90, 060301(R) (2014).

[63] D. Sels and A. Polkovnikov, Minimizing irreversible losses in quantum systems by local counterdiabatic driving, Proc. Natl. Acad. Sci. USA 114, E3909 (2017).

[64] T. Hatomura and K. Takahashi, Controlling and exploring quantum systems by algebraic expression of adiabatic gauge potential, Phys. Rev. A 103, 012220 (2021). 
[65] P. W. Claeys, M. Pandey, D. Sels, and A. Polkovnikov, Floquet-Engineering Counterdiabatic Protocols in Quantum Many-Body Systems, Phys. Rev. Lett. 123, 090602 (2019).

[66] N. Qian, On the momentum term in gradient descent learning algorithms, Neural Networks 12, 145 (1999).

[67] J. Duchi, E. Hazan, and Y. Singer, Adaptive subgradient methods for online learning and stochastic optimization, J. Mach. Learn. Res. 12, 2121 (2011).

[68] J. Dean, G. Corrado, R. Monga, K. Chen, M. Devin, M. Mao, M. a. Ranzato, A. Senior, P. Tucker, K. Yang, Q. Le, and A. $\mathrm{Ng}$, Large scale distributed deep networks, Advances in Neural Information Processing Systems 25 (NIPS, 2012).

[69] S. Ruder, An overview of gradient descent optimization algorithms, arXiv:1609.04747.

[70] S. G. Brush, History of the Lenz-Ising model, Rev. Mod. Phys. 39, 883 (1967).

[71] E. Ising, Beitrag zur Theorie des Ferromagnetismus, Z. Phys. 31, 253 (1925).

[72] E. Lifshitz and L. Pitaevskii, Statistical Physics: Part 2: Theory of the Condensed State, Course of Theoretical Physics Vol. 9 (Butterworth-Heinemann Ltd, Oxford, 2013).

[73] D. Leibfried, M. D. Barrett, T. Schaetz, J. Britton, J. Chiaverini, W. M. Itano, J. D. Jost, C. Langer, and D. J. Wineland, Toward Heisenberg-limited spectroscopy with multiparticle entangled states, Science 304, 1476 (2004).

[74] V. Giovannetti, S. Lloyd, and L. Maccone, Quantum-enhanced measurements: Beating the standard quantum limit, Science 306, 1330 (2004).

[75] C. L. Degen, F. Reinhard, and P. Cappellaro, Quantum sensing, Rev. Mod. Phys. 89, 035002 (2017).

[76] S. Choi, N. Y. Yao, and M. D. Lukin, Quantum metrology based on strongly correlated matter, arXiv:1801.00042.

[77] B. Nachtergaele and R. Sims, Lieb-robinson bounds in quantum many-body physics, in Entropy and the Quantum, edited by R. Sims and D. Ueltschi, Contemporary Mathematics Vol. 529 (American Mathematical Society, 2010), pp. 141-176.
[78] W. W. Ho and T. H. Hsieh, Efficient variational simulation of non-trivial quantum states, SciPost Phys. 6, 29 (2019).

[79] K. Binder and A. P. Young, Spin glasses: Experimental facts, theoretical concepts, and open questions, Rev. Mod. Phys. 58, 801 (1986).

[80] D. Sherrington and S. Kirkpatrick, Solvable Model of a SpinGlass, Phys. Rev. Lett. 35, 1792 (1975).

[81] M. P. Harrigan, K. J. Sung, M. Neeley, K. J. Satzinger, F. Arute, K. Arya, J. Atalaya, J. C. Bardin, R. Barends, S. Boixo, M. Broughton, B. B. Buckley, D. A. Buell, B. Burkett, N. Bushnell, Y. Chen, Z. Chen, B. Chiaro, R. Collins, W. Courtney et al., Quantum approximate optimization of non-planar graph problems on a planar superconducting processor, Nat. Phys. 17, 332 (2021).

[82] E. Farhi, J. Goldstone, S. Gutmann, and L. Zhou, The quantum approximate optimization algorithm and the SherringtonKirkpatrick model at infinite size, arXiv:1910.08187.

[83] T. Jörg, F. Krzakala, J. Kurchan, A. C. Maggs, and J. Pujos, Energy gaps in quantum first-order mean-field-like transitions: the problems that quantum annealing cannot solve, Europhys. Lett. 89, 40004 (2010).

[84] M. M. Wauters, R. Fazio, H. Nishimori, and G. E. Santoro, Direct comparison of quantum and simulated annealing on a fully connected Ising ferromagnet, Phys. Rev. A 96, 022326 (2017).

[85] M. Filippone, S. Dusuel, and J. Vidal, Quantum phase transitions in fully connected spin models: An entanglement perspective, Phys. Rev. A 83, 022327 (2011).

[86] T. Caneva, R. Fazio, and G. E. Santoro, Adiabatic quantum dynamics of a random Ising chain across its quantum critical point, Phys. Rev. B 76, 144427 (2007).

[87] Y. Seki and H. Nishimori, Quantum annealing with antiferromagnetic fluctuations, Phys. Rev. E 85, 051112 (2012).

[88] B. Seoane and H. Nishimori, Many-body transverse interactions in the quantum annealing of the $p$-spin ferromagnet, J. Phys. A: Math. Theor. 45, 435301 (2012).

[89] J. Wurtz and P. J. Love, Counterdiabaticity and the quantum approximate optimization algorithm, Quantum 6, 635 (2022). 